Aus der chirurgischen Universitätsklinik in Basel (Direktor Prof. Dr. F.de Quervain).

\title{
Zur Methodik der Magnesiumsulfatbehandlung des Tetanus.
}

Von Dr. Albert Wydler, Assistent der Klinik.

(Mit I Abbildung.)

Nachdem Kocher 1912 die von Meltzer experimentell begründete Magnesiumsulfat-Therapie als Erster bei 3 Tetanusfällen mit Erfolg angewandt hatte, konnte er $1914 / 15$ zu diesen 2 weitere günstig verlaufende Fälle hinzufügen. In seinen neuesten Mitteilungen ${ }^{2}{ }^{3}$ ) bespricht er ausführlich die Technik der Injektionen (siehe unten) und äußert sich mit $\mathrm{Me} 1 \mathrm{tzer}^{4}$ ) über die sowohl symptomatische als auch kurative Wirkung des ' $\mathrm{Mg} \mathrm{SO}_{4}$ bei Tetanus sehr zuversichtlich.

Gestützt auf zahlreiche experimentelle Untersuchungen stellten Meltzer und A u er die Maximaldosen des Magnesiumsulfats für die verschiedenen Injcktionsarten fest, und zwar $0,3 \mathrm{~g}$ pro $\mathrm{kg}$ Körpergewicht bei intralumbaler, I,5 $\mathrm{g}$ bei subkutaner, I,O $\mathrm{g}$ bei intramuskulärer Injektion. Intravenöse Injektionen erwiesen sich als sehr toxisch, so da $B$ sie von vornherein keine Anwendung fanden. Für den Menschen empfahlen die beiden Autoren vor allem die intradurale Injektion, und $\mathrm{Kocher}$ hat damit in der Tat bei 3 Fällen Erfolg gehabt. Die Technik war folgende: Lumbalpunktion, Ablassen einer entsprechenden Menge Liquor und Injektion von 2-10 $\mathrm{ccm}$ einer $15-25$ proz. Magnesiumsulfatlösung. Um die lähmende Wirkung derselben von der $\mathrm{Me}$ dulla oblongata fernzuhalten, wurde der Oberkörper des Kranken

1) Korr. Bl. f. Schw. Ärzte 1912, S. 969.

2) D. m. W. 1914, Nr. 46 u. 47.

3) Korr. Bl. f. Schw. Ärzte I9I 5, S. 1249.

4) B. kl. W. I9I 5 , Nr. Ir. 
etwas hochgelagert. Mehr als zweimalige Injektion pro die wurde vermieden, wegen Kumulationsgefahr.

Seither war es möglich, die Magnesiumsulfatwirkung auch bei den übrigen Applikationsarten zu studieren. Wegen ihrer Einfachheit fand zunächst die subkutane Injektion viele Anhänger. Es werden 20-30 proz. Magnesiumsulfatlösungen verwendet, und zwar hat die Erfahrung gezeigt, daß es nicht nötig ist, ist die Volldosis $(1,5 \mathrm{~g}$ per $\mathrm{kg}$ (Gewicht) auf einmal zu geben, sondern daß man sie auf 24 Stunden verteilen dürfe. Kocher injiziert als durchschnittliche Dosis $1 / 4$ der Volldosis, doch soll letztere in den ersten Tagen wenigstens in 24 Stunden erreiclit werden. Ganz analog der intralumbalen Injektion tritt die Wirkung nach 15-20 Minuten ein, ist aber von viel kürzerer Dauer. Einige ungünstig verlaufende Fälle, die an Kumulation zugrunde gingen ( $\mathrm{s}$ a d ler, W ydler), mahnten immerhin sehr zur Vorsicht und unterstützten die Bestrebungen die Injektionsart anzuwenden, die eine sichere Dosierung und eine unter Umständen sofortige Magnesiumwirkung gewährleistete. Es ist das Verdienst von $\mathrm{Straub}$, gezeigt $\mathrm{zu}$ haben, daß man bei Verwendung von verdünnten Lösungen ohne Gefahr vor toxischen Erscheinungen, in travenöse Infusionen machen kann, auch wenn man in refracta dosi sehr große Mengen Magnesiumsulfat einführen muß, da der Organismus den Anforderungen der Ausscheidung in weitgehendstem Maße gewachsen ist ( $\mathrm{Straub}$ ). Ich werde noch später Gelegenheit haben, näher auf die Dosierungen einzugehen.

Diese Erfahrungen der letzten Jahre veranlaßten Kocher zu der schon erwähnten zusammenfassenden Mitteilung in Nr. 40 des Korr.-Bl. f. Schw. Ärzte, I9 I5, in der er sehr bestimmt seine Schlußfolgerungen zieht, die ich hier folgen lasse:

,Wir können also jetzt viel bestimmter unsere Indikationen feststellen. bei Behandlung eines Tetanus, als wir dies in unseren früheren Publikationen getan haben. Denn durch unsere klinischen Beobachtungen sind wir zu der Einsicht gekommen:

I. Daß es nicht nötig ist, die von Meltzer und Auer für Erzielung einer völligen Muskelerschlaffung notwendige Dosis anzuwenden, sondern da $B$ es genügt, den Krampf zu heben, d. h. die Erregbarkeit der Zentren so weit herabzusetzen, daß unter Nachlaß 
der Starre die Anfälle aufhören - wenn auch ein gewisser Grad von Steifigkeit zurückbleibt.

2. Die zweite wichtige Tatsache klinischer Beobachtung ist die, daß bei der für genannten Zweck nötigen Dosis die Gefahr einer I.ähmung des Atemzentrums, oder allgemeiner gesprochen, eines Atemstillstandes, so gut wie nicht mehr vorhanden ist. Diese neuen Ergebnisse finden ihre Erklärung in dem Nachweis von $S t r a u b$, daß der Nachlaß der krampferzeugenden Überregung der Zentren früher eintritt, als die Lähmung der Frregbarkeit für die physiologischen Reize.

3. Der dritte Fortschritt, den die Beobachtungen am Menschen ergeben haben, ist der, daß eine crw ünschte Kumulation in der Wirkung rechtzeitig wiederholter Dosen besteht, und daß wir zuverlässige Anhaltspunkte haben für die Beurteilung des Grades der Kumulation. Die nötige Sättigung der Nervensubstanz, wie wir ohne Präjudiz über die Art der Magnesiumwirkung sagen können, ist nicht erreicht, so lange bei starker Muskelstarre anfallsweise Krampfsteigerung auftritt. Bei letzterer kann ohne Bedenken die Injektion wiederholt werden.

4. Das vierte Resultat der Beobachtung an tetanuskranken Menschen ist, da $B$ man bei Anwendung des Mittels in refracta dosi ganz gewaltige Mengen Magnesiumsulfat dem menschlichen Körper ohne irgendeinen Schaden einverleiben kann, weil das Medikament nach relativ kurzer Zeit ausgeschieden wird. Das ist der bedeutsame Vorteil dieses Mittels vor allen anderen. Die Zeit der Ausscheidung durch die Nieren geht parallel der Raschheit der Resorption; sie ist am: kürzesten nach intravenöser Zufuhr des Mittels, danach folgt dic intramuskuläre, dann die subkutane, und am längsten dauert die Ausscheidung nach intraspinaler Injektion. Dementsprechend ist die Kumulation am meisten $\mathrm{zu}$ berücksichtigen bei intraspinaler Applikation (noch nach 24 Stunden), danach bei subkutaner, dann intramuskulärer und zuletzt bei intravenöser Anwendung, wo sie schon nach $1 / 2-1$ Stunde kaum mehr ins Gewicht fällt.

Nach allen diesen Aufschlüssen sind wir gegenwärtig über die im Einzelfalle angezeigte Methode der Anwendung von Magnesiumsulfat viel besser orientiert als noch vor einigen Monaten und dadurch in der Lage, unter Beibehaltung der vollen Wirksamkeit des Mittels dessen Gefahren zu vermeiden.

Es kann keine Rede mehr davon sein, daß man - wie Cloet ta vorschlägt - die Magnesiumsulfatbehandlung des Tetanus auf die Fälle einschränkt, bei denen die Krämpfe auf die untere Körperhälfte (abwärts vom Brustmark) beschränkt sind. Das würde dieser sichersten aller kurativen Behandlungsmethoden den Hauptteil ihres Wirkungsgebictes entziehen. Im Gegenteil: Wo die Atenmuskulatur 
am Krampf mitbeteiligt ist, muB absichtlich auf dic krampfenden Atemmuskeln (bzw. ihre Nervenversorgung) gewirkt werden; bei subkutanen und intramuskulären Injektionen durch Steigerung der Dosen, bei intraspinaler Injektion durch Hochlagerung des Beckens und unteren Teiles des Thorax, bis die Starre des Thorax und der Rumpfmuskulatur nachläßt und die Atemkrämpfe ausbleiben.

Geht man von der Erreichung dieses Zieles ab, so gibt man unnütz gerade die schwcrsten 'Tetanusfälle preis, bei welchen das Magnesiumsulfat das zur Stunde einzig noch Hilfe bringende IIeilmittel ist.

Für die praktische Verwendung des Magnesiumsulfats ( $\mathrm{Mg} \mathrm{SO}_{4}$ c. aqua) läßt sich unser jetziger Standpunkt dahin zusammenfassen:

I. Das Magnesiumsulfat hat eine symptomatisch kurative Wir. kung, das Tetanusserum eine prophylaktische. Letzteres ist bei jedem Falle von Tetanusinfektion (durch Straßenkot, Garten- und Felderde usw.) verdächtiger $W$ unde sofort anzuwenden und alle 5, 8 und 12 Tage zu wiederholen. Es ist unnütz, über die durch Expcrimente für den Menschen festgestellte Dosis von $10 \mathrm{ccm}$ (Berner-Serum) in subkutaner Anwendung hinauszugehen.

2. Bei den Anfangssymptomen des Tetanus (gesteigerte Reflexerregbarkeit, Schluckweh, Schwierigkeit beim Essen, Steifigkeit) ist sofort eine subkutane Injektion einer 25 proz. Magnesiumsulfatlösung zu machen. Die Dosis muß nach dem Körpergewicht festgestellt werden. Die Maximaldosis beträgt $\mathrm{I}, 5 \mathrm{~g}$ per Kilo. Die durchschnittliche Dosis braucht nicht mehr als $1 / 4$ der Volldosis zu betragen; letztere mul3 aber in der Regel durch viermalige Wiederholung in 24 Stunden erreicht werden (in den ersten Tagen).

3. Der Patient muß genau und beständig überwacht werden behufs Einhaltung des richtigen Momentes der Wiederholung der Injektion. Dieser wird angezeigt durch Auftreten von anfallsweisen Krämpfen, in der Regel von Zunahme ider tonischen Starre begleitet.

4. Bei ungenügender Wirkung der subkutanen Anwendung des Sulfats, d. h. bei schweren Fällen, darf dic intramuskuläre Injektion benutzt werden. Die Dosis für dieselbe ist $\% / 3$ der subkutanen. Die Wirkung der intramuskulären Injektion geht rascher vorüber (wic sie rascher eintritt), muß deshalb eventuell sechsmal wiederholt werden, wo subkutan viermal (in 24 Stunden) genügen.

5. Für Fälle, welche mit stärksten Anfällen, die meist auch gehäuft auftreten, in Behandlung kommen, besonders bei Beteiligung der Atemmuskulatur mit Cyanose, muß anfänglich energischer vorgegangen werden, wenn man den Kranken retten will. Jeder schwere Anfall bedroht das Leben und ist durchaus zu verhüten. 
6. Drei Methoden zu raschester und kräftiger Wirkung sind dazu geeignet:

a) S tr a u b s intravenöse Injcktion. Sie löst die Krämpfe binnen I-- $;$ Minuten bei Benutzung einer 2,5 proz. Lösung vollständig, wenn binnen 2 Minuten $100 \mathrm{ccm}$ einflieben.

Schon nach EinflicBen von $25 \mathrm{ccm}$ der Lösung hat Stra ub beim erwachsenen Menschen die Krämpfe verschwinden sehen (also nach $21 / 2 \mathrm{ccm}$ der $25 \mathrm{proz}$. Lösung, bzw. $0,62 \mathrm{~g} \mathrm{Mg} \mathrm{SO} 4$ ). Für Erziclung einer Dauerwirkung muB dic Injektion halbstündlich bis stündlich wiederholt werden. (Die Kanüle bleibt in der Vena cubitalis eingebunden.)

Meltzer verwirft die intravenöse Methode wegen der Gefahr für das Herz.

b) Die Meltzer-Aucrsche Kombination von $/$ thernarkose mit intramuskulärer Magnesiumsulfatinjektion: Erst gute Äthernarkose (nicht Chloroform!), bald darauf Injektion von $\mathrm{MgSO}_{1}$ im Verhältnis von 0,5 per Kilo intramuskulär $=2 \mathrm{ccm}$ einer 25 proz. Lösung pro kg. Äthernarkose nach 20 Minuten fortgesetzt.

Weiterbehandlung mit $\ddot{A}$ therinhalation bei Wiedereintritt von Krämpfen, gefolgt je nach Grad der Wirkung von intramuskulärer, oder bloß subkutaner Injektion des 25 proz. $\mathrm{Mg} \mathrm{SO}_{4}$.

7. Die Straubsche und Mcltzer-Auersche Mcthode (Nr. 6a und b) ergeben keine große Gefahr einer Atemlähmung. Tritt sie ein, so injiziert man intramuskulär ( $\mathrm{Mel} \mathrm{tzer}$ ) eine 2 proz. isotonische Chloralcalciumlösung $\left(\mathrm{CaCl}_{2}\right)$ zu $; 0-60 \mathrm{ccm}$ intramuskulär oder eine 0,05 proz. Lösung in physiologischer Kochsalzlösung ( 6 Promille $\mathrm{NaCl}$ ) intravenös, bis die Atmung sich wieder einstellt, eventl. bis zu $600 \mathrm{ccm}$.

8. c) Dic intraspinale Injektion ist die Mcthode, welche bei den kleinsten Dosen den sichersten und anhaltendsten Erfolg erreicht. Sie wird gegen den Opisthotonus in kurzer Äthernarkose gemaclit. Die Dosis beträgt nach $\mathrm{Meltzer} 0.25 \quad \mathrm{MgSO}_{\text {f }}$ pro Io kg Gewicht $=\mathrm{I} \mathrm{ccm} 25$ proz. Lösung. Sie darf bei Kindern nicht ausgeführt werden wegen der Schwierigkeiten künstlicher Atmung und eventucll öfterer Wicderholung der Injektion.

Der Körper mit Extremitäten und Rumpf soll ganz horizontal gelagert werden, bloB der Kopf (nicht der Hals) von einem Kissen unterstützt, und zwar für mehrere Stunden. Tritt aber die krampflösende Wirkung für die Rumpf-, speziell Thoraxmuskulatur nicht ein, dann muß der Kopf horizontal gelagert werden und, wenn nötig, der ganze Körper schräg kopfabwärts.

MuB man dieses tun, so muB man gewärtigen, daß eine tiefe Narkose eintritt, die viele (bis 24) Stunden anhalten kann. Diese Narkose ist eine schr erwünschte Beigabe, aber sie ist mit der Möglichkeit eines rasch eintretenden Atemstillstandes verbunden. 
Letztere Möglichkeit verlangt gebieterisch, daß man bei intraspinaler Injektion den Patienten stetig überwache und mit neben dem Bette bereit gehaltenem Apparat sofort die künstliche Lungenlüftung nach Meltzer einlcite, mit unserer Modifikation, statt Luft Sauerstoff zuzuführen. Wir haben keinen einzigen unserer Patienten an dem Atemstillstand verloren; er bedingt also in Wirklichkeit keine Gefahr, da das IIerz absolut nicht geschädigt wird.

Zur Stunde, wie auch $M$ eltzer angibt, darf nach einer crsten wirksamen intraspinalen Injektion der Versuch einer Fort. setzung der Behandlung mit subkutanen oder intramuskulären Injektionen gemacht werden."

Hiernach erscheint die Frage als ziemlich gelöst. Richtige Dosierung und Technik vorausgesetzt, hält Ko ch e r Mißerfolge für selten. Wir werden später sehen, wieweit unsere Fälle mit Obigem übereinstimmen, und wo sie davon abweichen.

Auch an unserer Klinik wurde seit 1912 bei allen Tetanusfällen $\mathrm{Mg} \mathrm{SO}_{4}$ injiziert. Bis Oktober I9I 5 gelang es uns jedoch nicht, auch nur einen einzigen Fall durchzubringen. Ob dabei örtliche Ursachen eine Rolle spielen, und die Tetanusinfektion von Basel und Umgebung eine besonders schwere ist, kann vielleicht an Hand eines größeren Materials nach dem Kriege entschieden werden. $\mathrm{Da} B$ für die Häufigkeit und vielleicht auch für die Virulenz der Infektion regionäre und klimatische Faktoren mit in Betrachit kommen, das ist auch durch die Kriegs. erfahrungen nahegelegt worden. Bemerkenswert ist ferner, daß (nach mündlicher Mitteilung) der Tetanus im Engadin schr selten ist, und eine ähnliche Beobachtung machte auch Prof. de Quervain während I 5 jähriger Beobachtungszeit auf dem Hochplateau des Jura. Sicher ist jedenfalls das eine, daß unsere Mißerfolge nicht cinfach falscher Technik zur Last gelegt werden können, stimmt doch das von uns geübte Vorgehen ziemlich genau mit den Vorschriften von Meltzer, Kocher und S t a dle ${ }^{5}$ ) überein. Es ist überhaupt besser, die Bezeichnung ,falsche Technik" nicht zu benutzen, so lange sich die Frage noch im Versuchsstadium befindet und das „Richtige“ noch in diametral entgegengesetzten Richtungen gesucht wird.

Wir wollen nun gerade der Mißerfolge wegen suchen, an Hand unserer Fälle die Wirkungsweise des $\mathrm{MgSO}_{4}$ im mensch-

5) Stadler: B. kl. W. 1914, Nr. I, 3 u. 4. 
lichen Körper zu studieren und durch eine strenge Kritik der klinischen Symptome die Faktoren ausfindig zu machen, die zu den negativen Resultaten geführt haben.

Ich lasse zunächst unsere Fälle chronologisch geordnet folgen :

Fall I. Th. H., Schüler, I4 Jahre alt.

Anamnese: Früher, abgesehen von den gewöhnlichen Kinderkrankheiten, immer gesund. Am 6. XI. I912 verletzte sich Patient mil einer Gartenschaufel während der Arbeit am linken Schienbein. Der Vater legte einen Verband mit Airolgaze an. In den nächsten Tagen floß die Wunde immer etwas, machte aber sanst keine weiteren Beschwerden. Am I3. XI. fühlte sich Patient nicht wohl, klagte über Kreuzschmerzen, Kiefersperre und Nackensteifigkeit. Der Vater konsulticrte einen Arzt, welcher den Patienten am I4. XI. in die Klinik cinwies.

St a tus: Für sein Alter großer und kräftig entwickelter Patient von gutem Ernährungszustande und freiem Sensorium. Gewicht $40 \mathrm{~kg}$. Temperatur 37,4, Puls 96, voll, kräftig. Ausgesprochener Trismus und Kiefersperre. Nacken-, Rücken-, und Bauchmuskeln' sowie die der Extremitäten in tonischer Starre. Bei Geräuschen und Berührungen werden kleinere Zuckungen des ganzen Körpers ausgelöst. Crin: weder Eiweiß noch Zucker.

Lokalstatus: Am linken Unterschenkel unterhalb der Knicscheibe eine ca. $6 \mathrm{~cm}$ lange bis auf $1 \mathrm{~cm}$ epithelisierte Wunde mit schmierigen Granulationen.

Diagnose: Tetanus traumaticus.

Therapic: Excision der Wunde gut im Gesunden, ausgiebige Desinfektion mit Jodtinktur, Verband mit Perubalsam. Injektion voln Tetanusantitoxin, fo $\mathrm{AE}$, in die Lmgebung der Wunde. Isolierzimmer. Gegen Abend Zunahme der Stöße an Zahl und Intensität. Die Kiefersperre ist absolut geworden, Patient kann auch nicht mehr schlucken, er erhält 2 Kochsalzinfusionen, Morphium mehrmals 0,0 , für die Nacht 5,o Chloral, worauf .Patient bald einschläft und bis zum Morgen ziemlich Ruhe hat.

15. XI. Morgens Temperatur 39.1, Puls I40. Da gegen Mittag die Krämpfe häufiger werden, entschließt man sich, I2 Uhr, zur Lumbalpunktion. Es werden $2 \mathrm{ccm}$ Liquor abgelassen, hierauf I ccm Magnesiumsulfat, Io proz. Lösung, injiziert. Bald darauf Nachlassen der Krämpfe sowic der Muskelstarre. Patient trinkt und schluckt gut. $6 \mathrm{Uhr}$ nachmittags a us voller $\mathrm{R}$ uhe plötzlich heftiger Krampfantall, der insofern atypisch ist, als die Atmung nicht aussetzt; sie ist nur rasch und stertorös. Heraktion sehr beschlcunigt, Puls kaum fühlbar. Sofortige subkutane Physostigmininjcktion $(0,0003)$ und Finleitung der Chlorofornarkose. Erschlaffen 
sämtlicher Muskeln. Die Atmung wird wieder tief und ruhig. Kochsalzinfusion. Um weiteren Krampfanfällen vorzubeugen, wird, da gegen 9 Uhr wieder leichte Muskelsteife auftritt, erneut die Lumbalpunktion ausgeführt. Ablassen von $2 \mathrm{ccm}$ Liquor, Injektion von $2 \mathrm{ccm}$ Io proz. $\mathrm{MgSO}_{4}$-Lösung. Patient schläft bald darauf ruhig ein.

I6. XI. Da im Laufe des Morgens Patient wieder unruhig wird und kleine Stöße auftreten, erneute Lumbalpunktion, Injektion von $2 \mathrm{ccm} \mathrm{MgSO}_{4}$-Lösung, Io proz., nach Ablassen von $2 \mathrm{ccm}$ I.iquor. Puls 38,7 Temperatur I I4. Patient hat während des ganzen Tages keine weiteren Anfälle, trinkt ruhig, löst spontan Urin, schläft am Abend mit o,OI Morphium ruhig ein.

17. XI. 2,40 nachm. Großer Krampfanfall von Io Minuten Dauer. Patient cyanotisch, atmet nicht mehr. Puls I46, klein. Sofortige künstliche Sauerstoffinsufflation mit dem Überdruckapparat plus Chloroformnarkose. Die Atmung wieder ruhig, Muskeln erschlafft. Noch während der Narkose Injektion von $2 \mathrm{ccm}$ ro proz. $\mathrm{MgSO}_{4}$ Lösung, intralumbal; Patient wieder schlaff, ohne Krampfanfälle. 4.40 morgens a us Ruhe plötzlicher grober Anfall und Exitus an Herzstillstand, trotz Sauerstoffinsufflation, Coffein und Chlorcaliumlösung 5 Proz., intravenös.

Sektionsprotokoll: Tetanus traumaticus.

$10,5-1,5 \mathrm{~cm}$. Großer Hautdefekt am linken Unterschenkel.

Status: Thymo-lymphaticus. Oedema pulmon. incipiens. Oedema pulmon. incipiens.

Herz: Etwas groß, linker Ventrikel fest kontrahiert. Epicard überall glatt und glatt. In den Höhlen reichlich Cruor und Speckhaut. Venöse Ostien für 2 Finger durchgängig. Endocard überall glatt und glänzend, Klappen zart, Aortaumfang $5 \mathrm{~cm}$ zart und glatt. Pulmonalis $6 \mathrm{~cm}$ ebenfalls zart und clastisch. Coronargefäße durchgängig mit glatter, zarter Wand. Wanddicke links Io, rechts 4. $; \mathrm{mm}$. Muskel transparent. Trabekel kräftig, Ventrikel nicht erweitert.

Cervicale Lympdrüsen markig geschwellt. In den großen Halsvenen Blutgerinnsel. Linke Gaumenmandel stark hypertrophisch. Iymphdrüsen am Hilus weich, geschwollen, stark antracotisch.

Lunge: Linke Pleura außer einigen Verwachsungen an der Spitze glatt, Bronchien ziemlich stark gerötet. Rechte Lunge etwas vermehrtes Volumen. Schleimhaut und Bronchien beiderseits gerötet, einzelne enthalten etwas gelbliche schleimig eitrige Flüssigkeit. Gefäße zart.

$S c h \ddot{d}$ eld a ch sy m m ctris ch. Diploe stark entwickelt. Dura glatt und spiegelnd, löst sich leicht von der Schädeldecke. Sinus longitud. enthält etwas Gerinnsel. Pia zart, Gefäße zicmlich stark mit Blut gefüllt. IIirnsubstanz feucht, Gyri normal entwickelt, Gefäße an 
der Hirnbasis zart. Verteilung von grauer und weißer Substanz normal. An den großen Ganglien keine pathologischen Veränderungen. Sinus der Schädelbasis enthalten etwas flüssiges Blut. Rückenmark: Äußerlich keine Besonderheiten.

Mikroskopisch im Rückenmark keine Veränderungen.

Knochenmark des rechten Oberschenkels rot, nur im untern Drittel cin etwa $5 \mathrm{~cm}$ langes Stück Fettmark.

Fall 2. A. K., $3 \mathrm{r}$ Jahre, Gärtner.

A namnese: Am 22. VII. IgI3 stürte Patient mit scinem Velo auf der Straße und zog sich dabei eine Verletzung seines linken Knies zu. Die stark verunreinigte Wunde wurde vom Arzte mit $\mathrm{H}_{2} \mathrm{O}_{2}$ gespült und nach Einlegen einer Vioformgazemeche verbunden. Sie heilte gut per granulationem. Am 3I. VII. hatte Patient Schluckbeschwerden und leichte Kiefersperre. am 2. VIII. Schmerzen und Steifigkeit im Nacken, am 3. VIII. auch in den Muskeln des Rückens, Bauches und der Extremitäten. Der Arzt schickt Patienten ins Spital.

Status: Grazil gebauter Patient von mäßigem Ernährungs. zustand und freiem Sensorium. Gewicht $60 \mathrm{~kg}$. Ausgesprochener Trismus. Kiefersperre, Opisthotonus. Brettharte Spannung der Bauchdecken, Temperatur 37,4. I'uls 8o. Urin: weder Eiweiß noch Zucker.

Lokalstatus: L'ber der linken Patella eine halbmondförmige weitklaffende, granulierende Wunde, ca. $5 \mathrm{~cm}$ lang, wenig sezcrnierend. L'mgebung der Wunde nicht gerötet. Inguinaldrüsen leicht vergrößert, nicht druckempfindlich.

Diagnose: Tetanus traumaticus.

Ther a pie: Toilette der Wunde, Anlegen einer breiten Gegenincision, ausgicbiges Ätzen mit konzentrierter Karbolsäure, nach'dem man auf Agar und Bouillon abgeimpft hatte. Tetanusantitoxin, 4o AE. subkutan in die Umgebung der Wunde. -

In der Bouillon anaerob gezüchtet, massenhaft Tetanusbazillen. Eine Maus, die damit greimpft wurde, geht nach 15 Stunden an Tetanus zugrunde.

Verla uf: Für die Nacht des 3. VIII. I9r 3 mehrmals Morphium o, O I sowic 5.0 Chloral. Patient schläft verhältnismäßig ruhig bis zum Morgen.

4. VIII. Mäufige, kleine Stöße, bei jeder Berïhrung und kleinsten Bewegungen des Paticnten. Patient erhält alle 3 Stunden o,oo I Koniin und schläft damit regelmäßig cin. 'Temperatur am Abend 38,0 , Puls 108, für die Nacht Chloral 4,0, worauf Patient ruhig cinschläft.

5. VIII. Da sich die Stöße von Stunde zu Stunde mehren und intensiver werden, entschließt man sich, um dem Patienten Ruhe zu verschaffen, zur Lumbalpunktion. Injektion von $2 \mathrm{ccm}$ 
einer 25 proz. $\mathrm{MgSO}_{4}$-Lösung, nach Ablassen von $2 \mathrm{ccm}$ Liqour ( 8 Uhr vorm.). Nach kurzer Zeit Nachlassen der Anfälle, Muskulatur schlaff, Patient schluckt wieder gut, und hat keine Anfälle während des Tages, schläft die ganze Nacht nach Verabreichung von Koniin o,oor sowie Chloral 4,o bis zum Morgen.

6. VIII. Da die Krämpfe wieder auftreten (I8-20 in der Minute) und sich zunehmende Muskelsteifigkeit bemerkbar macht, gibt man wieder eine $\mathrm{MgSO}_{4}$-Injektion, $2 \mathrm{ccm}$. 25 proz. Lösung, wie oben. Patient ist während des Tages ziemlich ruhig, schläft viel. Atmung ruhig. $30-36$ pro Min. (Puls 100-114, Temperatur hält sich zwischen 38 und 39 Grad. I I Uhr morgens a u s'völliger R uhe plötzlich ein groBer StoR, von 31/2 Min. Dauer. Patient pulslos, hoch cyanotisch, atmet nicht mehr. Sofortige Sauerstoffinsufflation mit Überdruckapparat und II crzmassage. Subkutan Physostigmin 0,0003 und Kampfer. Nach kurzer Zeit Puls wieder fühlbar, nach 15 Minuten erster spontaner Atemzug. Da Patient nach dem Anfall ruhig schläft, sicht man vorläufig von einer weiteren $\mathrm{MgSO}_{4}$. Injektion $a b$.

7. VIII. Im Laufe des Morgens fast keine 7uckungen; Trismus und Opisthotonus bestehen noch, doch fühlt sich Patient ordentlich, kann gut schlucken und stillt seinen Durst mit Tee. Gegen Mittag wieder häufigere kleine Zuckungen, so da $B$ man nachmittags $2 \mathrm{Uhr}$ cine erneute $\mathrm{MgSO}_{4}$-Injektion macht, $2 \mathrm{ccm}$ einer $25 \mathrm{proz}$. $\mathrm{MgSO}_{4}$-Lösung nach Ablassen von $5 \mathrm{ccm}$ Liquor (I.iquordruck erhöht). Nach kurzer Zeit Nachlassen der Muskelstarre und der Anfälle. Es besteht nur noch etwas Nackensteifigkeit, Temperatur 38, I, Puls 100. Morphium o,or. Patient schläft abends ruhig ein.

8. VIII. 2,25 nachm. Aus $\mathrm{ruhigem} \mathrm{Schlafe}$ cin kleincr, kurzer Stoß von einigen Sekunden, $\mathrm{mit} \mathrm{schwerem} \mathrm{Herz}$. kollaps; Patient atmet noch. Intravenös sofort Chlorcalcium 5 Proz., $5 \mathrm{ccm}$; Herzmassage sowie Sauerstoffinsufflation ohne Erfolg.

Sektion 8. VIII. Anat. Diagnose:

Tetanus traumaticus.

Lappenwunde am linken Knie.

Stauung sämtlicher Abdominalorgane.

Subseröse Blutungen in Pleura-, Perikardial- und Peritonealhöhle.

Sukmuköse Blutungen im Magen und Duodenum.

Meckelsches Divertikel.

I\} e r z: Entsprechend groß. Subepikardiales Fett reichlich. Konsisten $z$ links gut, rechts etwas schlaff. Venöse Ostien für 2 Finger durchgängig. In den Höhlen viel Speckhaut und flüssiges Blut. Mitralis mit geringgradiger Verdickung am Schließungsrande. Aortenklappen zart, Aortaumfang $61 / 2 \mathrm{~cm}$, Intima zart. Tricuspidalis mit geringgradigen Verdickungen am Schließungsrande. Pulmonal- 
klappen zart, ebenso Pulmonalis. Umfang $7 \mathrm{~cm}$. Ventrikel nicht erweitert. 'Trabekel, Papillarmuskeln kräftig. Endokard zart. Myokard braunrot, gut transparent. Wanddicke links II, rechts $4 \mathrm{~mm}$. Coronargefäße zart, Foramen ovale geschlossen.

Lungen: Von mittlerem Volumen und gutem Luftgehalt. Stellenweise subpleurale kleine Blutaustritte. Pleura glatt und glänzend. Bronchienschleimhaut leicht gerötet.

Ma gen: Mit schleimigem Inhalt. An einigen Stellen submukös strichförmige, dunkle, frische Blutungen. Schleimhaut des Duodenums ebenso.

H irn: Nahtsubstanz spärlich. Diploe reichlich, dunkel. Dura von mittlerer Spannung. Durainnenfläche glatt und glänzend. Durale Sinus mit viel flüssigem Blut. Weiche Häute glatt und spiegelnd, zart. PIexus stark bluthaltig. Gefäße der Basis zart. Seitenventrikel nicht erweitert. Ependym zart. 3. Ventrikel o. B.. ebenso 4. Ventrikel. Die Hirnsubstanz gut durchfeuchtet, mit einer großen Anzahl von Blutpunkten. Stammganglien. Pons, Medulla oblong. und Kleinhirn o. B.

R ückenmark: Etwas hyperämisch, sonst o. B.

Mikroskopisch: Rückenmarkshyperämie, sonst keine Veränderungen.

Fall 3. H. Sch., I 3 Jahre, Schüler.

A namnese: Strauchelte am 3. II. IgI4 auf der Straße und zog sich eine kleine Wunde an der Vola manus sin. zu; dieselbe fing in den nächsten Tagen an zu eitern, doch schenkte Patient derselben keine weitere Aufmerksamkeit und ging, ohne ärztliche Behandlung, täglich in die Schule. Am 12. II. klagte Patient über Steifigkeit im Nacken, konnte den Mund nicht mehr gut öffnen und hatte heftige Kreuzschmerzen. Erst jetzt wurde ein Arzt konsultiert, welcher am I2. II. den Patienten in die Klinik schickte.

Status: Mittelgroßer, gut entwickelter Patient, von gutem Ernährungszustand und freiem Sensorium. Gewicht $42 \mathrm{~kg}$. Trismus, Kiefersperre, Opisthotonus, bretthartc Bauchdeckenspannung, Steifigkeit in den Extremitäten. Schlucken ist möglich, doch treten dabei sofort Krämpfe auf, die für den Patienten sehr schmerzhaft sind. Innere Organe o. B.

Lokalstatus: In der Vola manus sin. eine $3 \mathrm{~cm}$ lange, tiefe, sezernierende Wunde. Umgebung derselben infiltriert und Wundränder leicht gerötet und druckempfindlich. Hand sowie Finger in starker Beugekontraktur. Axillardrüsen geschwollen und etwas druckempfindlich.

Puls 100, voll, regelmäßig. Temperatur 37,8 .

Diagnose: Tetanus traumaticus.

Therapie: Toilette der Wunde in leichtem Ätherrausche. Es werden dünne Holzsplitter und Papierfetzchen aus der Tiefe 
der Wunde entfernt und auf Agar und Bouillon, überimpft. (Es wachsen massenhaft Tetanusbazillen; geimpfte Mäuse gehen nach 24 Stunden an typischen Krämpfen zugrunde.) Die Wunde selbst wird erweitert und ausgiebig mit dem Paquelin verschorft; breite Tamponade mit Vioformgaze. In die Umgebung der Wunde subkutane und intramuskuläre Injektion von Tetanusantitoxin. 6o A E. Isolation des Patienten.

Verla uf: Zur Bekämpfung der Spasmen wird eine subkutane Injektion von $\mathrm{Mg} \mathrm{SO}_{4}$-Lösung 30 Proz., $2 \mathrm{ccm}$, gegeben, ohne allen Erfolg, weshalb man nach 2 Stunden eine weitere Injektion, $3 \mathrm{ccm}$, macht, zugleich 3,0 Chloral per Clysma. 20 Minuten später weichen die Spasmen, die Muskulatur erschlafft, und Patient schläft bald ruhig ein. Da gegen Mitternacht wieder vereinzelte kleine Stöße auftreten und die Muskulatur spastisch wird, gibt man wieder $\mathrm{Mg} \mathrm{SO}_{4}$, 3o proz. Iösung, 3,0 subkutan, worauf Patient bis zum Morgen ruhig schläft.

I3. II. Gegen 8 Uhr vorm. kehrt die Muskelstarre wieder zurück; kleinere und größere Stöße in rascher Folge. Erneut $\mathrm{MgSO}_{4}$, 30 Proz., 6,0 subkutan und da ohne den gewünschten Erfolg, 2 Stunden später intramuskulär 3,o. Frst jetzt weichen die Spasmen ganz, die Muskulatur ist schlaff, Patient nimmt Flüssigkeit zu sich und schläft dann bis $4 \mathrm{Uhr}$ nachm. Wegen neuen heftigen Krämpfen Wiederholung der $\mathrm{Mg} \mathrm{SO}_{4}$ - Injektion und zwar subkutan 6,0 und da wieder ohne große Wirkung, intramuskulär 3,0, cine Stunde später. Erst jetzt erschlafft die Muskulatur wieder und Paticnt schläft bis zum Abend. - Temp. 40,3, Puls kräftig, voll, I32. Patient nimmt viel Flüssigkeit zu sich, außerdem erhält er eine subkutane Infusion. Katheterismus wegen Retentio urinae. Nachts 12 Uhr Muskulatur wieder spastisch, kleine, häufige Zuckungen; eine $\mathrm{Mg} \mathrm{SO}_{4}$-Injektion, 6,0, subkutan, 30 Proz., bringt diesmal bald Erschlaffung. Patient hat Ruhe bis zum Morgen.

14. II. Temp. 39.5, Puls 120 , etwas kleiner, aber regelmäßig. Koffein und Kampfer. Zugleich wieder $\mathrm{MgSO}_{4}$, 30 proz., 3, $\mathrm{O}$ subkutan. Patient ist vollkommen schlaff. 12 Uhr 45 Min. nachm. plötzlich aus voller Ruhe, bei schlaffer Muskulatur, großer StoB, mit Herz- und Atcmstillstand. Sofort intravenös Chlorcalcium, 5 proz., 5,0. Physostigmin 0,0003; Sauerstoffinsufflation mit Überdruck, da nach $1 / 2$ Stunde kein Erfolg Tracheotomie und direkte Insufflation, sowie direkte Herzmassage nach Thorakotomic; auch diese crfolglos.

Sektionsdiagnose: Rißwunde an der linken Vola manus.

Tetanus traumaticus.

Status nach Tracheotomie, Freilegung des Herzens zur Herzmassage.

Käsige Bronchialdrüsen-Tuberkulose. 
Herz: Entsprechend groß, Spitze vom linken Ventrikel gebildet. Subepikardiales Fett spärlich, Konsistenz links wenig, rechts deutlich herabgesetzt. Venöse Ostien für einen Finger durchgängig. Höhlen fast leer. Klappenendokard zart, ebenso Kranzarterien, Aorta und Pulmonalis. Umfang von Aorta und Pulmonalis $5 \mathrm{~cm}$. Wanddicke links 9, rechts $2 \mathrm{~mm}$. Myokard braunrot, transparent. Papillarmuskel und Trabekel kräftig. Ventrikel nicht erweitert. Foramen ovale geschlossen.

Lungen: Frei, von mittlerem Volumen, Konsistenz beidseits etwas vermehrt, Gewebe überall von geringem Luft- und starkem Blutgehalt. Pleurahöhlen leer.

Magen: o. B.

Gehirn und Rückenmark: o. B.

Fall 4. O. S., 28 Jahre, Handlanger.

An amnese: Immer gesund. Verletzte sich am I4. II. I9I4 mit einem Messer $z$ wischen Daumen und Zeigefinger. Ein Arzt nähte I- 2 Stunden später die Wunde, die jedoch nicht ganz heilte. Am 28. II. hatte Patient Schmerzen in der linken Schulter, die er für rheumatische hielt. Erst als am x. III. Kiefersperre und Nackensteifigkeit auftrat, suchte er am 2. III. den Arat auf, der ihn ins Spital schickte.

St a tus: Mittelgroßer, grazil gebauter Patient von mäßigem Ernährungszustande und freiem Sensorium. Deutlicher Trismus, Opisthotonus; Bauchmuskulatur etwas gespannt, Extremitäten frei beweglich. Atmung ruhig, regelmäBig. Herz o. B. Puls 76 , voll, kräftig. Temp. 37,2. Patellarreflexe gesteigert. Urin weder Eiweiß noch Zucker.

Lokalstatus: Zwischen Daumen und Zeigefinger der linken Hand eine $4 \mathrm{~cm}$ lange, zum Teil per primam geheilte Schnittwunde, deren radialer Winkel von atrophischen Granulationen eingenommen wird. Die Sonde kommt nirgends in die Tiefe. Axillardrüsen nicht geschwellt.

Therapic: Excision der Wunde im Gesunden und nachfolgende Verätzung mit Jodtinktur. Subkutan und intramuskulär in dic Umgebung der Wunde Tetanusantitoxin $40 \mathrm{AE}$. Isolation des Patienten, Morphium.

Die Nacht vom 2./3. III. verläuft ruhig. Patient hat keine Krämpfe und schläft meistenteils.

3. III. Zunahme der allgemeinen Muskelstarre im Laufe des Tages, weshalb eine subkutane $\mathrm{MgSO}_{4}$-Injektion, 3,0, 30 Proz. Lösung, gemacht wird. Da ohne große Wirkung, nach einer Stunde Wiedcrholung derselben und zwar 6,0. Darauf allgemeine Erschlaffung. Patient verfällt auch bald in einen ruhigen Schlaf, der bis zum Morgen anhält. Tempcratur am Abend 38,3. Puls 80.

4. III. Muskelstarre wieder allgemein, kleine Zuckungen mit heftigen Schmerzen und starkem Schweiß. $\mathrm{MgSO}_{4}, 30$ Proz, 6,o 
subkutan, :worauf bald wieder vollständige Erschlaffung eintritt. Patient trinkt und ißt. Am Abend wegen wieder eintretender Muskelstarre und Krämpfen nochmals 6,0 $\mathrm{MgSO}_{4}$ 30 Proz., womit Pat. die ganze Nacht schläft. Temp. 39, I. Puls 120.

5. III. Morgens einzelne Zuckungen, Muskelstarre. $\mathrm{MgSO}_{4}$ 30 Proz., 6,0 subkutan, sowie Tetanusantitoxin, 40 AE. in die Umgebung der Wunde. Pat. nach $1 / 2$ Std. schlaff. Im Laufe des Nachmittags treten wieder kleine Krämpfe auf, mit leichter Dyspnoc, daher $\mathrm{MgSO}_{4} 30$ Proz., 6,o subkutan, ganz ohne alle Wirkung, deshalb 2 Stunden später die gleiche Dosis wiederholt. Temp. 39,8. Puls I4O. Nach $1 / 2$ Stunde Pat. schlaff, schläft ruhig ein.

6. III. 9 Uhr vorm. großer tonisch-klonischer Anfall, über den Patient mit Sauerstoffinsufflationen gut hinübergebracht wird. Z:1gleich subkutan 6,0 $\mathrm{MgSO}_{4} 30$ Proz., worauf Patient wieder ruhig atmet. Puls etwas schwach, daher Srophantininjektion I promillige Lösung intravenös. Im Verlaufe des Morgens plötzlicher Herzkollaps. Digalen, $\mathrm{CaCl}$, 5 Proz., Herzmassagre, Sauerstoffinsufflation können den Tod nicht verhindern.

Sektionsdiagnosc: Tetanus traumaticus. Lungenödem. Hypostase im linken Unterlappen.

Herz: Vergrößert, beidseits schlaff, Spitze abgerundet. Im linken Ventrikel kleine, hellrote Blutungen, in den Höhlen flüssiges Blut. Venöse Ostien für 2 Finger durchgängig. Klappen nur spurweise verdickt. Aortaumfung $6 \mathrm{~cm}$, mit kleinen trüben Flecken. Kranzarterien zart, ebenso Pulmonalis. Umfang $7 \frac{1}{2} \mathrm{~cm}$. Ventrikel etwas erweitert, Trabekel. Pap. Muskel abgeplattet. Links ein Tawarascher Sehnenfaden. Wanddicke links I I, rechts $3 \mathrm{~mm}$. Muskel blaß braunrot und von guter Transparenz. Foramen ovale geschlossen.

I. u n g e n: Groß, Pleura glatt spiegelnd. Auf Schnitt, beide Oberlappen und der rechte 'Mittellappen hellgraurot, gut lufthaltig, kompressibel, g-latt und glänzend. Der linke Unterlappen dunkelrot, sehr blutreich, feucht, stellenweise brüchig und leicht gekörnt. Unterlappen rechts ebenfalls blutreich, gut lufthaltig mit reichlich blutig schaumiger Flüssigkeit. Pulm. art. zart, Bronchien gerötet. An den rechtsseitigen Bronchien bis in die feinsten Verzweigungen schmierige bräunliche Massen. Bronchialdrüsen groß, blutreich.

$G$ eh irn: Durainnenfläche glatt und spiegelnd. Im Sinus longitudinalis flüssiges Blut. Gehirn äußerlich o. B. Rückenmark makroskopisch und mikroskopisch ohne Veränderungen.

F all 5. F. G. 38 Jahre, Gärtner.

A n a m nese: Verletzte sich vor 3 Wochen infolge Anschlagen an der großen Zehe. Am 2. XI. I9I4 konnte Patient sich kaum mehr fortbewegen, da seine Beine steif waren, den Mund konnte Patient nur noch wenig öffnen; auch hatte er beim Versuch etwas zu trinken heftige Schlingkrämpfe. Eintritt ins Spital 2. XI. I9I4. 
St a t u s: Sehr kräftig gebauter, mittelgroßer Patient, sehr guter Ernährungszustand, Körpergewicht $78 \mathrm{~kg}$ etwas Habitus aetylicus, freies Sensorium. Ausgesprochener T'rismus, Kiefersperre. Opisthotonus und tonische Starre der unteren Extremitäten sowie der Bauchmuskeln. Innere Organe: Herz o. B.

Lungen: Emphysem, leichte Bronchitis.

Lrin: o. E. o. Z. Temperatur 37,2, Puls 96.

Reflexe kaum erhöht.

Lokalstatus: Linke grobe Zehe sowie zweite und dritte weiscn alte Rißwunden auf mit eitriger Sekretion und fötidem Geruch, gringe Verfärbung. Inguinaldrüsen nicht akut vergrößert.

Diagnose: Tetanus traumaticus.

Fxartikulation der 1. 2. und 3. Zehe, letanusantitoxin in die Limgebung der Wunde, so A E. Offene Wundbehandlung.

Verla uf: Im Laufe des Nachmittags tritt leichte Nackenstarre auf, die: Bauchmuskulatur von Zeit zu Zeit bretthart gespannt. Mit Morphium 0,02 und 4,0 Chloral hat Patient eine ziemlich ruhige Nacht.

3. XI. Im Laufe des Morgens treten cinzelne, kleine Stöße auf, 2- 4 in der Stunde, 9 Uhr morgens ein größerer Anfall mit allgemeiner Körperstarre und I)yspnoc; daher eine subkutane $\mathrm{MgSO}_{4}$ Injektion, i 5 Proz., 10,o. Die Krämpfe bleiben nun aus, kehren jedoch schon gegen I $\mathrm{Uhr}$ nachm. zurück, weshalb man eine neue $\mathrm{MgSO}_{4}-\mathrm{In}$. jektion I 5 Proz. 20,0 macht. Auch diesmal die Wirkung wieder von sehr kurzer Dauer, da die Krämpfe schon gegen Abend 5 Uhr wied:r zurückkehren und die Muskelstarre allgemein wird.

Die Temperatur 38,5 , Puls 1,2 , klein, unregelmäßig, wiederholt Digalen und Kampfer; da die Krämpfe bedrohlichen Charakter annehmen tracheotomiert man und gibt abends $8 \mathrm{Uhr}$ eine weitere MgSO -Injektion, subkutan 30 Proz., 6,0. Bald Erschlaffung und Ausbleiben der Krämpfe, zugleich wird die Herzaktion schlechter und schlechter, obschron Patient ruhig atmet unter Zufuhr von Sauerstoff. 12 Uhr nachts wieder Auftreten von Krämpfen, daher $\mathrm{MgSO}_{4}$ 60,25 Proz. Lösung.

4. XI. 6 Whr morgens wieder ein großer Anfall; Sauerstoffinsufflation. Irerzmassage, Digalen, Kampfer. Physostigmin 0,0003 Patient erholt sich, ist wieder vollkommen schlaff, atmet ruhig; 7 Uhr plotalich Exitus an II e c z tillstand a u $\beta$ erhalbeines Anfalles.

An at. Diagnose: Status nach Amputation von 3 Zehen des rechten fußes und nach Tracheotomie.

Particlle Lungenatelektase. Chron. Lungenemphysem. Stauung der Abdominalorgane. Fettleber.

I. un gen von geringem Volumen, in den Oberlappen besonders reichlich emphysematös, in den untern Partien fast luftleer. Die

Deutsche Zeitschrift f. Chirurgie. $13^{6}$. Bd. 
Gewebe wenig bluthaltig, kompressibel. flaumig. Bronchien mit etwas Schleim. Schleimhaut ziemlich stark injiziert. Pulmon. Gefäße zart.

H e r z: Von entsprechender Größe, Konsistenz links gut, rechts gering. Spitze vom linken Ventrikel gebildet. Venöse Ostien für 2 Finger durchgängig. Ilerzhöhlen mit reichlich flüssigem Blut. Klappein sämtlich zart. Aorta ascendens und Arteria pulmon. von mittlerem Lmfang ( 7 bzw. $8 \mathrm{~cm}$ ). Intima zart, I erhöhlen nicht erweitert, Wanddicke links I I, rechts $4 \mathrm{~mm}$. Myokard braunrot, transparent. Coronargefäße zart, Foramen ovale geschlossen.

Ma gen: Schleimhaut hyperämisch, etwas verdickt, ebenso im Duodenum.

G ehirn: Diploe reichlich. Dura mater und weiche IIäute zart, o. B. Gehirn weist nur geringe Hyperämie auf, sonst keine Besonderheiten.

Rückenmark zeigt auffallend starke Injektion in den dorsalen Arterien und Stauung in den ventr. Venen.

Mikroskopisch: Leber weist sehr weite Zentralvenen auf. ziemlich ausgedehnte Verfettung der Leberzellen.

Niere mit sehr starker Hyperämie zwischen den geraden Kanälchen und auch in den Glomeruli.

Fall I und 2 wurden intralumbal, Fall 3,4 und 5 subkutan, zum Teil kombiniert mit intramuskulären Injektionen behandelt. Sämtliche Fälle müssen wir mit Rücksicht auf die klinischen Erscheinungen zu den schweren bis sehr schweren Tetanusfällen rechnen; bei Fall $\mathrm{I}-3$ stimmt damit auch die kurze Inkubationszeit von 7,8 und 9 Tagen überein.

Die Magnesiumwirkung auf den Muskeltonus war bei allen Fällen eine ausgezeichnete. 15-30 Minuten nach der Injektion waren die Kranken vollkommen schlaff und erinnerten in nichts mehr an das noch vor kurzem so schwere Krankheitsbild. Ein Unterschied zwischen intralumbaler und subkutaner Injektion hinsichtlich des Eintrittes der Magnesiumwirkung bestand nicht, nur hinsichtlich der Dauer derselben dürfte die intralumbale die subkutane und intramuskuläre Injektion übertreffen. Sie betrug nämlich bei ersterer I2-I4 Stunden, bei letzterer 7-Io Stunden, obschon die verabreichte Magnesiummenge ein Vielfaches der bei intralumbaler Injektion verwendeten betragen muß. Die Dosierung beträgt bei intralumbaler Anwendung 0,03 g per kg Körpergewicht, bei subkutaner $1,5 \mathrm{~g}$, bei intramuskulärer ungefähr $2 / 3$ letzterer, also $\mathrm{r}, \mathrm{O} \mathrm{g}$, und das ist für den Organismus, namentlich aber für ein durch das 
Tetanustoxin geschwächtes Herz, nicht gleichgültig. Einen weiteren Vorteil der intralumbalen Injektion sahen wir auch darin, daß die gewünschte Erschlaffung immer prompt eintrat, während, wie Fall 3 und 4 zeigen, bei subkutaner Injektion dieselbe gelegentlich ohne Erfolg war und darum wiederholt werden mußte. Dic Beantwortung dieses Phänomens ist keine schwere und kann durch das Tierexperiment schön demonstriert werden: Ein Kaninchen, auf einen Tisch gebunden, erhält verschiedene subkutane $\operatorname{Ig~} \mathrm{SO}_{4}$-Injektionen, welche auch nach längerer Zeitdauer keine Narkose bewirken. Sobald nun das Tier abgebundeil wird, stirbt es nach wenigen Minuten in $\mathrm{Mg} \mathrm{SO}_{4}$-Narkose an Niemlähmung (Stra u $\left.{ }^{6}\right)$ ), mit anderen Worten, durch die Bewegungen des Tieres wurde die Resorptionsgeschwindigkeit geändert, so daß plötzlich tödliche Dosen des $\mathrm{Vg} \mathrm{SO}_{4}$ in den Kreislauf kamen, welche Atmungs- und Herzzentrum lähmten.

Diese inkonstante Resorptionsgeschwindigkeit aus den Geweben ist der Grund, weshalb sowohl bei intralumbaler als auch subkutaner Injektion die Losicrung eine so unsichere ist, und sie erklärt uns auch die schon von andern Autoren (Stadler) beobachteten Kumulationserscheinungen des Magnesiums. Gerade unsere Fälle 2, 4 und 5 sind typische Beispiele dafür. Zwei sterben außerhalb eines Anfalles bei schlaffer Muskulatur und freier Atmung an Herzlähmung, welche mit $\mathrm{Ca} \mathrm{Cl}_{2}$ nicht mehr rückgängig gemacht werden kann. Fall 2 geht während eines kleinen nur wenige Sekunden dauernden Infalles an Herzstillstand zugrunde. Es ist kaum denkbar, daB dieser durch Asphyxie zustande kam, denn Patient atmete noch einige Minuten ruhig weiter.

Inwiefern bei Fall $\mathrm{I}$ und 3 die $\mathrm{Mg} \mathrm{SO}_{4}$-Kumulation an dem letalen Ausgange, der ebenfalls durch Herzstillstand erfolgte, eine Rolle spielt, ist nicht sicher zu entscheiden. Hier scheint mir, handelt es sich mehr um eine $\mathrm{Kumulation} \mathrm{der} \mathrm{Te-}$ tan ustoxinwirkung. Deshalb sehen wir bei beiden Kranken, die unter der Magnesiumwirkung ruhig atmen und völlig schlaff daliegen, plötzlich und ohne vorhergehende $A$ zeichen, mit elementarer Gowalt auftretende Anfälle.

6) D. In. IV. 1915, Nr. I. 
Das ist eine Beobachtung, wie sie meines Wissens noch nicht beschrieben worden ist. Sie bildet zusammen mit den unerwünschten Nebenwirkungen des Magnesiums eine, weitere, die Therapie sehr erschwerende, Komplikation. Bisher machten wir, mit andern Autoren, die Indikation von neuen $\mathrm{Ig} \mathrm{SO}_{4}$-Injektionen abhängig von dem Nachlassen der Magnesiumwirkung, die sich durch Auftreten von gesteigerter Reflexerregbarkeit und Zurückkehren der Muskelstarre geltend machte. Jetzt jedoch befanden wir uns in einem Dilemma. Einesteils kannten wir die sehr geringe Narkotisierungsbreite des $\mathrm{Mg} \mathrm{SO}_{4}{ }^{7}$ ), und riskierten durch prophylaktische Injektionen diese zu überschreiten und Kuml. lation hervorzurufen, andernteils waren wir nie sicher, auch bei schlaffem Körper plötzlich von einem großen Anfall überrascht zu werden.

Wir stehen vor der Wahl von zwei Übeln, welche beide gleich groß sind: Kumulation des Magnesiums und paroxysmale Steigerung der Toxinwirkung. Wir werden uns deshalb nach einer Injektionsweise umsehen müssen, welche uns erstens vor Kumulationserscheinungen des $\mathrm{Mg} \mathrm{SO}_{4}$ schützt, und uns' zweitens in die Lage versetzt, plötzlich auftretende große Anfälle mit Magnesium sofort zu unterdrüken. In Betracht kommt, wie es mis scheint, einzig die intravenöse Injektion. Diese darf jedoch nicht in der hohen Konzentration wie bei der subkutanen Injektion zur Verwendung kommen, da schon relativ kleine Dosen

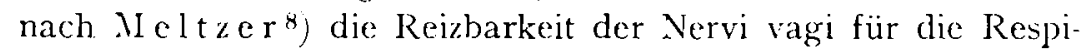
ration herabsetzen und Atmungsstillstand hervorrufen. St $r$ a ub ${ }^{y}$; hat deshalb nach zahlreichen experimentellen Untersuchungen an Tieren vorgeschlagen, intravenöse Infusionen von 3 proz. $\mathrm{MgSO}$-Lösungen zu geben. Als mittlere gerade noch wirksame Einflußgeschwindigkeit gibt er $100-150 \mathrm{ccm}$ in der $\mathrm{Mi}$ nute an. Mit einer solchen Dosis gelang es ihm auch am Menschen immer vollständige Erschlaffung zu erzielen. Diese betrifft vor allem die in Krampf befindliche Muskulatur, welche jedoch nicht vollständig, sondern nur bis zu ihrer normalen Funktionsfähigkeit gelähmt wird. Die Wirkung tritt nach der

7) Issekutz: Ther. M. H. 1915, Juli.

8) B. kl. IV. 1906, S. 76 .

9) M. m. W. 1915, Nir. 10. 
Injektion fast momentan auf, und diese kann durch die in eine Armvene eingebundene Dauerkanüle zu jeder Zeit leicht wiederholt werden.

Durch analytische Bestimmung der im Harn in 24 Stunden ausgeschiedenen Salze konnte $\mathrm{Str}$ a ub auch beweisen, da $B$ der Organismus den Anforderungen der Magnesiumausscheidung in weitgehendem Maße gewachsen ist, so da $\beta$ wir also bei intravenöser Injektion der $\mathrm{Mg} \mathrm{SO}_{4}$-Salze vor Kumulationserscheinungen ziemlich sicher sind. Sollten trotzdem solche auftreten, so sind wir durch die Dauerkanüle zu jeder Zeit in der Lage, mit dem Antidot des $\mathrm{Mg} \mathrm{SO}_{4}$ dem $\mathrm{CaCl}_{2}$ die Magnesiumwirkung sofort aufzuheben.

Ich habe nun vor kurzem Gelegenheit gehabt, einen 'Tetanusfall mit intravenösen Infusionen zu behandeln und mich ron der Richtigkeit der Straubschen Experimente und Angaben zu überzeugen. Da ich bis jetzt in der Literatur keinen so behandelten Fall ausführlicher beschrieben finde, teile ich den unsrigen in extenso mit:

Fa 11 6. N. A., 68 Jahre, Landarbeiter. Spitaleintritt 17 . XI. 19I5 mittags I 2 Uhr.

An a m nese: Patient früher immer gesund, leidet seit ca. 20 Jahren an doppelseitigen Inguinalhernien und wird heute mit der Diagnose: : Hernia incarcerata in die chirurgische Klinik eingewiesen. Er gibt an, daß3 er seit letzten Samstag I3. XI. I9I 5 die sonst leicht reponible Hernie nicht mehr reponieren könne. Der Bruch sei auch im ganzen jetzt vicl größer und gespannter anzufühlen als gewöhnlich. Wind und Stuhlabgang normal, kein Erbrechen, doch gibt Patient an. daß3 er in den letzten Tagen ziehende Schmerzen im Kreuz und im Bauch verspürt habe und dab er beim Schlucken große Beschwerden habe (1;. XI.). Von einer Verletzung oder einem Unfall will Patient nichts wissen.

St atus: Mittelgroßer, grazil gebauter Patient, von mäßigem E. Z., ca. $50 \mathrm{~kg}$ schwer. Ausgesprochener Risus sardonicus sowie starke Kiefersperre. Zunge stark belegt und trocken. Pupillen gleich weit, mittelweit, reagieren sehr wenig. Der Kopf ist in die Kissen eingebohrt und kann nicht nach vorne gebeugt werden.

Atmung vollkommen frei, 20 in der Minute. Lungen: über briden Spitzen eine ausgesprochene Dämpfung und über beiden Unterlappen bronchitische Erscheinungen.

H e rz: in normalen Grenzen, Töne rein, Aktion regelmäßig. P'uls 92, voll, kräftig; Temperatur 37,6. A bdomen bretthart 
gespannt. Bei der Palpation desselben treten kleine, kurzdauernde Anfälle des ganzen Körpers auf. Links besteht eine gut zweimannsfaustgroße Hernia inguino-scrotalis. I)ieselbe ist stark gespannt, läßt sich nur mit Mühe reponieren und tritt bei Freilassen der für 3 Finger gut durchgängigen Bruchpforte infolge der starken Bauchdeckenspannung sofort wieder aus. Auf der rechten Seite besteht eine zweite, ca. gänsecigroße Hernia inguinalis directa.

Die untern Extremitäten befinden sich in tonischem Kuntraktionszustand. Dic Patellarreflexe sind beidseits stark gesteigert. Die Arme sind beweglich, doch etwas fixiert. Die Lendenwirbelsäule stark kyphotisch.

Eirne genaue Revision des Körpers ergibt nirgends eine frische Wunde noch Narbe. Irüsenschwellungen sind keine vorlanden. Urin: weder Eiweiß noch Zucker.

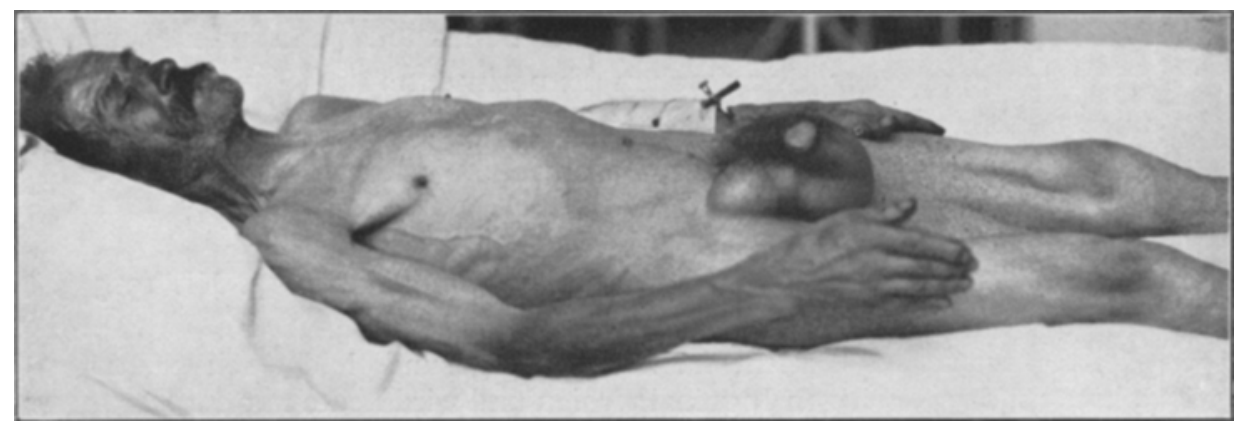

Fig. 1.

Diagnose: Tetanus.

Patient erhält 0,01 Mo schläft, nur ron kleinen Zuckungen von Zeit zu Zeit geweckt, den größten Teil des Nachmittags. Da gegen Abend die Zuckungen sich merklich häufen und schon bei kleinen Geräuschen im Zimmer ausgelöst werden, wird abends $6 \mathrm{Lhr}$ nach Einbinden einer paraffinierten Kanüle in eine Armvene eine intravenöse Infusion von $\mathrm{MgSO}_{4} 3$ proz. Lösung $100 \mathrm{ccm}$ gemacht mit einer Einflußgeschwindigkeit von 60 Sekunden. Da dieselbe ohne den gewünschten Erfolg ist, wird sie 7,10 Uhr wiederholt, zugleich injiziert man auch $40 \mathrm{AE}$ Tetanusantitoxin subkutan und o.or Mo. Auch diesmal ist die Wirkung des Magnesiums nur eine partielle, die tonische Starre der Muskulatur läßt schon etwas nach und die Krampfanfälle sind etwas weniger häufig, aber ganz schlaff ist der Patient nie. 8,30 Uhr muB Patient katheterisiert werden $900 \mathrm{ccri}$ Urin. Gegen 12 Uhr nachts nimmt die Muskelstarre wieder zu, die Zuckungen sind sehr häufig und von längerer Dauer. Man 
macht deshalb eine erneute Infusion $100 \mathrm{ccm} 3$ proz. $\mathrm{MgSO}_{4}$-Lösung. Ebenfalls mit nur teilweisem Erfolge.

18. XI. 1915. Von 4 Uhr morgens an ist Patient unruhiger und hat oft kleine Zuckungen. Der Körper ist absolut starr. 9 Uhr Infusion einer 5 proz. $\mathrm{MgSO}_{4}$-Lösung 100,0 und als auch jetzt wieder die Erschlaffung keine vollständige ist und die Krampfanfälle trotzdem auftreten, wiederholt man dieselbe 12,40 Uhr, und zwar $120 \mathrm{ccm}$ einer 5 proz. $\mathrm{MgSO}_{4}$-Lösung bei einer Linflußgeschwindigkeit von 60 Sekunden. Nach ca. I Minute erfolgt bei starker Cyanose plötzlich Atemstillstand. Das Herz schlägt ruhig weiter, die Aktion ist etwas verlangsamt, 76 . Sofort künstliche Atmung mit dem 1 berdruckapparat und eine intravenöse Injektion einer 5 proz. $\mathrm{CaCl}_{2}$ lösung 50:0. Nach weiteren 2 Minuten erfolgt wieder der erste Atemzug. Im übrigen ist jetzt Patient ganz schlaff und hat keine Anfälle mehr bis 4,15 $\mathrm{Uhr}$ nachm. Tracheotomie superior. (5 $\mathrm{Vhr}$ abends.) Bei allgemeiner Muskelstarre und häufig auftretenden Lirampfanfällen Injektion von $60 \mathrm{ccm}$ ciner 5 proz. $\mathrm{MgSO}_{4}$-Lösung bei einer Einflußgeschwindigkeit von 20 Sekunden. Nach $3+$ Minuten Körper absolut schlaff, keine Krämpfe mehr. Patient schläft hald ruhig ein, erhält 8,30 Uhr $0,2 \mathrm{~g}$ Luminal. Von 9 Uhr ab macht sich wieder eine leichte Starre in den untern Extremitäten und am Bauch bemerkbar. Gegen io Uhr treten auch wieder einzelne kleinerc Zuckungen auf bei freier Atmung und ruhigem Puls.

I9. XI. I9I5. Wegen allgemeiner Starre und häufigen Anfällen 12,20 vorm. intravenöse Injektion von $70 \mathrm{ccm}$ einer 5 proz. $\mathrm{MgSO}_{4^{-}}$ I.ösung. Körper nach $31 / 2$ Minuten ganz schlaff, keine Krämpfe mehr, Patient schläft ruhig ein, nachdem er spontan Urin gelöst hat. Der ganze Morgen ist ruhig und Patient bleibt bis auf die Kiefer-und Nackenstarre ziemlich schlaff, verlangt des öfteren zu trinken. Tem. peratur 37,3. Puls I12. Gegen 12 Uhr mittags wieder einige kleine kurzdauernde Stöße und zunehmender Muskeltonus. Eine intravenöse Injektion von $30 \mathrm{ccm} 5 \mathrm{proz} . \mathrm{MgSO}_{4}$-Lösung bringt nach 3 Winuten wieder vollständige Erschlaffung. Während des Nachmittags schläft Patient meistens, zwischen 3 und 4 kehrt wieder ein leichter Muskeltonus zurück, Anfälle jedoch treten nicht auf. Patient hat leichte Atemnot, welche jedoch mit Sauerstoffinsufflation leicht gehoben wird. $7 \mathrm{Uhr}$ intravenöse Kochsalzinfusion $\mathrm{I} / 2$ Liter, subkutan 20 A $\mathrm{E}$ Tetanusantitoxin. Für die Nacht 0,2 Luminal, welches auf den Patienten sehr beruhigend wirkt. Während der Nacht keine Krämpfe, dic Muskulatur im allgemeinen schlaff, nur noch leichte Kiefersperre und Nackenstarre.

20. XI. Temperatur 37,2, Puls, 100, voll, kräftig. Patient im allgemeinen schlaff, Kiefersperre und Vackenstarre weniger intensiv, I0,30 Uhr 20 A E Tetanusantitoxin. $\Lambda \mathrm{m}$ Abend Temperatur 37, $\$$ Puls 92. Atmung ruhig. Patient hat große Mühe zu expekto- 
rieren, so daB am $2 \mathrm{I}$. XI., da die Tetanussymptome mehr und mehr verschwinden und Patient bereits sitzen kann, das Dekanülement rorgenommen wird. 23. XI. Temperatur zwischen 37 und 38 , Atmung sehr oberflächlich beschleunigt, rostfarbenes Sputum, Patient leicht cyanotisch. 24. XI. Temperaturanstieg bis 38,5 . Heration regelmäßig, Puls jedoch schwächer. Z̈ber beiden Unterlappen eine ausgesprochene Dämpfung, verstärkter Stimmfremitus, Bronchialatmen. Unter zunehmender Herzschwäche erfolgt am 25. Xl. mittags 12 Uhr der Exitus letalis.

Pathologisch anatomische Diagnose: I obäre Pncumonic beider Lnterlappen, rernarbte Spitzentuberkulose beidseits. I) ilatatio cordis, Myocarditis chronica, Pleuritis adhaesiva links, chronisches Lungenemphysem. Atrophie der Milz und der Nieren, Stauungs- und Fettleber.

Rückenmarkshäute zeigen mäBige Hyperämie, Rückenmarksubstanz makroskopisch und mikruskopisch o. B., ebenso auch das Gehirn. in den Venen des Armes nirgends 'Thrombusbildung nachweisbar.

Nach den klinischen Symptomen, der allgemeinen Körperstarre, den sehr häufigen Zuckungen müssen wir, obschon wir die Inkubationsdauer nicht ermitteln können, den Fall zu den mittelschweren rechnen. Wo die Injektionspforte zu suchen ist, konnten wir weder aus der klinischen Cintersuchung noch aus der Autopsie entscheiden. Interessant ist jedenfalls die 'Tatsache, daß unter Unständen schon kleine, makroskopisch kaum sichtbare Verletzungen der Haut oder der Schleimhäute als Infektionspforte in Betracht zu ziehen sind, obgleich dieselben sicher dem Tetanusbazillus die sonst im allgemeinen geforderten Lebensbedingungen, wie Mischinfektion, Sauerstoffabschluß, nicht bieten.

Zur Zeit, als wir den Tetanusfall erhielten, war schon alles Toxin an das Nervensystem gebunden. Wenigstens zeigten 4 mit verschieden großen Blutmengen gespritzte weiße Mäuse absolut keine Tetanuserscheinungen. Dennoch injizierte ich dem Paten am ersten Tag $40 A E$, am 3., 4. und 6. Tage je $20 \Delta \mathrm{E}$ Ietanusantitoxin, nicht in der Hoffnung, damit das schon gebundene Toxin zu neutralisieren, sondern um zufällig neugebildetes Toxin im Blute unschädlich zu machen. Um die Infusionen zu jeder Zeit rasch ausführen zu können, band ich nach der gewohnten Technik in irgendeine Armvene eine Glaskanüle ein, die mit einem kurzen, durch eine Klemme abgeschlossenen Gummischlauch armiert wurde (siche Iigur). Die Kanüle blieb 
mehrere Tage durchgängig, und wenn man noch die Vorsichtsmaßregel anwendet, dieselbe vor dem Einnähen durch Eintauchen in 45-5ogradiges Paraffin zu paraffinieren, ist man vor 'Thrombose und Embolie ziemlich sicher.

Anfänglich hielt ich mich mit der Iosierung genau an die Vorschriften von $\mathrm{Straub}$, d. h. ich machte Infusionen von I $00 \mathrm{ccm}$ einer 3 proz. $\mathrm{Mg} \mathrm{SO}_{4}$ - Lösung bei einer Einflußgeschwindigkeit von 60 Sekunden, ohne jedoch die Muskelstarre und die Krämpfe ganz unterdrücken zu können. Auch eine Infusion von I oo $\mathrm{ccm}$ einer 5 proz. Lösung bei gleicher Einflußgeschwindigkeit hatte nur partielle Wirkung. Daß ich mit dieser Dosierung aber nahe an der therapeutisch noch erlaubten Grenze war, zeigte dic nächste Injektion ron $\mathrm{I}_{20} \mathrm{ccm}$ der $5 \mathrm{proz} . \mathrm{Mg} \mathrm{SO}_{4}$ Lösung, die nach $2-3$ Minuten prompten Atmungsstillstand hervorrief, während das Herz ohne wesentliche Verlangsamung der Schlagfrequenz ( 76 gegenüber 88 früher) noch ruhig weiter schlug, also ganz in C̈bereinstimmung mit den Angaben Meltzers, nach denen Herzstillstand erst nach der vollständigen Lähmung der Atemmuskulatur erfolgt. Es besteht demnach hier cin prinzipieller Unterschied zwischen subkutaner und intralumbaler Injektion einerseits, intravenöser andererseits. Während jene durch Kumulation direkt Herztod verursachen können (Fall I - 5), scheinen diese zuerst zu Atmungsstillstand und erst sekundär zu Herzstillstand zu führen. Was für dic Therapie besonders wichtig ist: wir besitzen im $\mathrm{CaCl}_{2}$ ein sicher wirkendes Mittel, die Lähmung des Atmungszentrums sofort rückgängig zu machen, im Gegensatze zu den Zirkulationsstörungen, welche durch $\mathrm{Ca} \mathrm{Cl}_{2}$ nicht gebessert werden. So sehen wir nach der sofortigen intravenösen Injektion von $50 \mathrm{ccm}$ einer 5 proz. $\mathrm{CaCl}$-Lösung und künstlicher Atmung mit dem Überdruckapparat bereits nach 4 Minuten wieder den ersten spontanen Atemzug.

Dic bisherigen Infusionen der 3 proz. Lösung waren nicht imstande, totale Erschlaffung zu erzielen. Es lag wohl nicht an der Menge. sondern eher an der Konzentration bzw. Geschwindigkeit, mit der sie in den Kreislauf gelangte. Ich injizierte deshalb, als nach 4-5 Stunden die Körperstarre und die Krämpfe wieder zurückkehrten, mit einer großen Spritze $60 \mathrm{ccm}$ einer 5 proz. $\mathrm{Mg} \mathrm{SO}_{4}$ - Lösung so rasch als möglich. Schon nach 3 bis 
4 Minuten waren die Körperstarre und die Krämpfe wic weggeblasen und der Patient war völlig schlaff bis auf eine leichte Kiefersperre und Nackenstarre, die nicht ganz verschwanden. Die Wirkung dieser Injektion dauerte 4 Stunden, doch war eine Wiederholung derselben erst nach 6 Stunden nötig, wiederum mit dem Erfolge völliger Erschlaffung nach 2-3 Minuten, und einer Wirkungsdauer von 12 Stunden. Den gleichen Erfolg hatte auch eine Injektion von $30 \mathrm{ccm}$ der 5 proz. Lösung (um I 2 thr mittags am 19. November 1915). Von da ab traten die 'Tetanuserscheinungen mehr und mehr in den Hintergrund und 2 Tage später waren auch die letzten Symptome, die Kiefersperre und die Nackenstarre, vollständig verschwunden, so daß man praktisch den Tetanus als geheilt betrachten konnte. Leider stellen sich nachträglich Erscheinungen von seiten der Lungen ein. Patient hatte auch nach dem Dekanülement große Mühe zu expektorieren und erlag am 25. November einer P'neumonie beider Unterlappen. Inwieweit daran die Tracheotomie Schuld trägt, ist schwer zu entscheiden, immerhin wäre es wünschenswert, daß dieselbe bei älteren I,euten unterlassen werden könnte. Ich habe noch nachzutragen, daß das in den ersten 24 Stunden injiziertc Magnesiumsulfat vollständig in dem ausgeschiedenen Urin enthalten war.

Pathologisch-anatomisch wurden in keinem der unsrigen lälle, die 1914 am Berner Pathologischen Institute ${ }^{10}$ ) beobachteten Schädigungen der grauen Substanz des Rückenmarkes gefunden. Es scheinen demnach diese Veränderungen mehr der Grundkrankheit als dem $\mathrm{Mg} \mathrm{\textrm {SO } _ { 4 }}$ zugeschrieben werden zu müssen.

Wenn wir gestützt auf das von uns Beobachtete unsere Eindrücke zusammenfassen wollen, so müssen wir vor allem betonen, daß die Zahl der Beobachtungen viel zu klein ist, um bindende Schlüsse zu ziehen. Wie sehr solche kleine Statistiken dem Zufall unterworfen sind, das haben ja auch die Nitteilungen aus den Lazaretten reichlich gezeigt. Um nur eines zu erwähnen, so hat Prof, de Quervain mit Dr. Ad. Viseher im lazarett in Badenweiler während der ersten Kriegsmonate von Io Tetanusfällen 6 heilen schen. Nur einer, und zwar einer der gestorbenen hatte Magnesiumsulfat erhalten. Noch viel weniger

ı) Korr. Bl. f. Schw. Ärzte 1914, S. 725 . 
als aus den 5 ungünstigen wollen wir aus dem einen relativ günstigen Falle weitgehende Folgerungen ableiten. Immerhin schien uns die Vergleichung der verschiedenen Applikationsweisen des Magnesiumsulfates so belehrend, daß wir die gewonnenen Findrücke in einige Sätze zusammenfassen wollen. Dieselben lauten folgendermaßen:

I. Das Magnesiumsulfat ist bei der symptomatischen Behandlung des Tetanus ein sehr brauchbares, aber nicht ungefährliches Mittel. Ohne eine fortwährende genaue Überwachung des Patienten darf es nicht zur Anwendung kommen.

2. I)urch subkutane, intramuskuläre oder intralumbale Injektionen ist es fast immer möglich, die Muskelstarre und die Krämpfe zum Verschwinden zu bringen und für die Dauer von 7-I4 Stunden vollständige Erschlaffung zu erziclen.

3. Ije Dosierung ist bei den obengenannten Injektionsarten wegen der sehr inkonstanten Resorptionsgeschwindigkeit eine unsichere, so daß wir stets mit Kumulationserscheinungen des $\mathrm{Mg} \mathrm{SO} \mathrm{S}_{4}$ rechnen müssen, die zu Herzstillstand führen können.

4. Da die Wirkung des $\mathrm{MgSO}_{4}$ erst 20-30. Minuten nach der subkutanen Injektion eintritt, sind wir gegenüber der paroxysmalen Wirkung des 'Tetanustoxins, die sich in plötzlich aus vollster Ruhe auftretenden großen Anfällen äußert, machtlos, und prophylaktische Injektionen bei noch schlaffem Körper zu machen verbietet uns die Gefahr der Magnesium-Kumulation.

5. Es crscheint deshalb zweckmäßig, die subkutanen, intramuskulären und intralumbalen Injektionen durch intravenöse $\mathrm{zu}$ ersetzen. Da die Magnesiumausscheidung eine vollständige und rasche ist, ist die Gefahr der Magnesium-Kumulation bei dieser Anwendungsform eine sehr kleine und dank der sehr rasch eintretenden Wirkung der intravenösen $\mathrm{Mg} \mathrm{SO}$-Injektion können wir uns auch rasch gegen die paroxysmale Wirkung des Tetanustoxin verteidigen. Theoretisch könnte man glauben, daß dies nun wirklich die ,richtige Anwendungsweise“ sei, doch bedarf es einer viel größeren Zahl von Boobachtungen, um dies behaupten zu können.

Um dic Injektion möglichst rasch ausführen zu können, binden wir eine Glaskanüle in eine Armvene ein; dieselbe muß, da 
sie als Dauerkanüle funktionieren soll, zur Vermeidung ron Thrombosenbildung paraffiniert werden.

7. Line Dosis von $30-50 \mathrm{ccm}$ einer 5 proz. Magnesiumsulfatlösungr mit einer Spritze möglichst rasch injiziert, genügt in der Regel, um nach 2-3 Minuten Muskelstarre und Krämpfe für eine Dauer von 4-5 Stunden vollständig zu beseitigen.

8. Zu starke Magnesiumwirkung kann durch eine Injektion von $20-50 \mathrm{ccm}$ der 5 proz. $\mathrm{CaCl}_{2}$-Lösung sofort aufgehoben werden. Dieselbe sollte deshalb im .Momente der Magnesium. sulfatinjektion stets zur Benutzung bereitstehen. 